\title{
Multiphoton autofluorescence and second-harmonic generation imaging of the tooth
}

\author{
Min-Huey Chen \\ National Taiwan University Hospital \\ Taipei 100, Taiwan \\ and \\ National Taiwan University \\ College of Medicine \\ School of Dentistry \\ Taipei 100, Taiwan
}

\section{Wei-Liang Chen \\ Yen Sun}

Peter Tramyeon Fwu

Chen-Yuan Dong

National Taiwan University

Department of Physics

Taipei 106, Taiwan

\begin{abstract}
In this study, we used an epi-illuminated multiphoton microscope to image three main components of ex vivo human tooth. In particular, we obtained two-photon excited autofluorescence (AF) and second-harmonic generation (SHG) images of the enamel, dentin, and periodontal ligaments (PLs) and constructed three-dimensional projections of sequentially and axially acquired images. We found that the enamel has a strong two-photon AF signal, clearly revealing the structures of the enamel rods. The dentin, on the other hand, has both AF and SHG signals. The contrast provided by the combination of these two imaging modalities can be used to reveal the structure of peritubular dentin and to distinguish the less mineralized circumpulpal dentins. The SHG and multiphoton AF imaging also showed the structure of the $\mathrm{PL}$ and the distribution of cells around the $\mathrm{PL}$, respectively. For comparison, we also obtained scanning electron microscopy images of the enamel, dentin, circumpulpal dentin, and the PL. Our results demonstrate the effectiveness of using multiphoton microscopy to visualize the major constituents of teeth, including enamel, dentin, and the PL, and the potential of this minimally invasive technique for monitoring the morphological developments during tooth regeneration. $\odot 2007$ Society of Photo-Optical Instrumentation Engineers. [DOI: $10.1117 / 1.2812710]$
\end{abstract}

Keywords: multiphoton autofluorescence; second-harmonic generation (SHG); microscopy; dentistry.

Paper 06339R received Nov. 20, 2006; revised manuscript received Jun. 10, 2007; accepted for publication Jun. 29, 2007; published online Nov. 28, 2007.

\section{Introduction}

The development of an effective, minimally invasive imaging modality is invaluable for investigating a variety of physiological phenomena and pathological conditions in dentistry. Finding an effective and minimally invasive method for imaging the structures of teeth is invaluable in active areas of current dental research including tooth regeneration. The traditional method of studying calcified sections of tooth involved the cutting of the tooth with a diamond saw or carborundum disk. In such methods, the tooth specimens need to be ground and polished until they are sufficiently thin to be viewed with reflected or transmitted light. For years, the structure of enamel has been described and studied with these procedures. For a more detailed view of the structure of teeth, it is necessary to decalcify and stain the tooth specimens, which would likely alter the highly mineralized structures of the tooth. The limited light transmission due to the mineralization and tooth thickness decreased the effectiveness of using confocal microscopy to study dental structures. The application of SEM has increased the understanding of the dental structures. However, the preparation for scanning electron mi-

Address all correspondence to M. H. Chen, National Taiwan University Hospital, College of Medicine, Dept. of Dentistry, Taipei 100, Taiwan; Tel: +886-22312-3456; Fax: +886-2-2383-1346; E-mail: minhueychen@ntu.edu.tw, or C. Y. Dong, National Taiwan University, Dept. of Physics, Taipei 106, Taiwan; Tel: +8862-3366-5155; Fax: +8862-2363-9984; E-mail: cydong@phys.ntu.tw croscopy (SEM) examination is complicated. In recent years, multiphoton microscopy has become an important research tool in biological research. ${ }^{1-5}$ A number of advantages render multiphoton microscopy the preferred imaging modality in many biomedical applications. To begin with, the use of near infrared excitation allows deep sample penetration. In addition, unlike single photon fluorescence microscopy, the nonlinear fluorescence and harmonic generation of multiphoton microscopy produce a signal only at the focal point. The pointlike excitation feature has the benefits of causing less sample photodamage and achieving sectional images without the need of a confocal pinhole. Signal collection without a confocal pinhole also improves the detection efficiency because more of the sample scattered light can be detected. The characteristic autofluorescence (AF) from cellular constituents can provide both morphological and functional information. In addition, many noncentrosymmetric biological structures can be used to produce second-harmonic generation (SHG) signals. SHG is a coherent, nonlinear process that generates particularly strong signals when the molecules are orderly arranged. Biological structures such as fibril collagen, muscle fibers, and microtubules are known to be effective in generating second-harmonic signals. ${ }^{6-9}$ Among tissues capable of generating second-harmonic signals, collagen is a particularly

1083-3668/2007/12(6)/064018/6/\$25.00 @ 2007 SPIE 


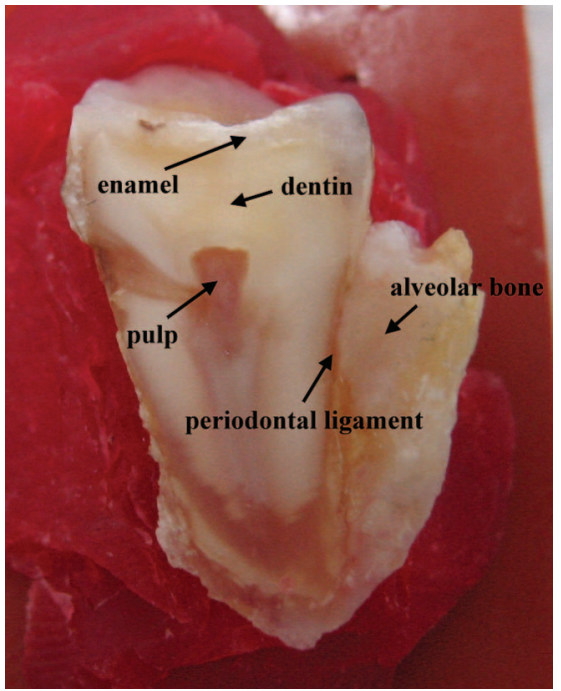

Fig. 1 Photo of the longitudinal section of the permanent secondary premolar used in this study. Structures of the enamel, dentin, pulp, PL, and alveolar bone attached to the root surface are indicated.

interesting subject of study due to its wide distribution in various tissues.

To apply multiphoton microscopy in research areas such as tooth development (odontogenesis) and dental tissue engineering, it is important to test the ability of multiphoton imaging in resolving the various structures of a fully grown tooth. The main components of teeth include the enamel, dentin, pulp, and the periodontal ligaments (PLs). The enamel is the most visible part of a tooth. It is highly mineralized to provide the strength to withstand the force of mastication and to protect the dentin. The dentin consists of mostly collagen and forms a channel structure called a dentinal tubule that radiates from the pulp to the enamel and cementum. Dentin is formed by the odontoblasts located in the dental pulp close to dentin. This process of dentinogenesis occurs throughout one's life span.

Obtaining the structural information of teeth is important for the study of dental development and regeneration. The dental pulp is at the center of teeth where blood vessels, nerves, and odontoblasts are located. The PLs are collagen fibers that hold a tooth in place by connecting the cementum to the jaw bone. PLs are produced and destroyed by the fibroblasts that are located throughout the ligaments and can be categorized according to their orientation. ${ }^{10}$

Although multiphoton imaging has been previously applied to the study of dental structures including dental carries, ${ }^{11-14}$ our work uses multiphoton microscopy to reveal both the two- and three-dimensional microscopic structures of enamel and dentin as well as that of the PL without the decalcification treatment of the tooth. We also compare our results with the structures acquired with SEM in an attempt to demonstrate that the images obtained from multiphoton microscopy serve as effective structural models for the investigation of teeth.

\section{Materials and Methods}

The tooth sample used in this study was obtained from a 30 -year old adult patient who had the upper left secondary
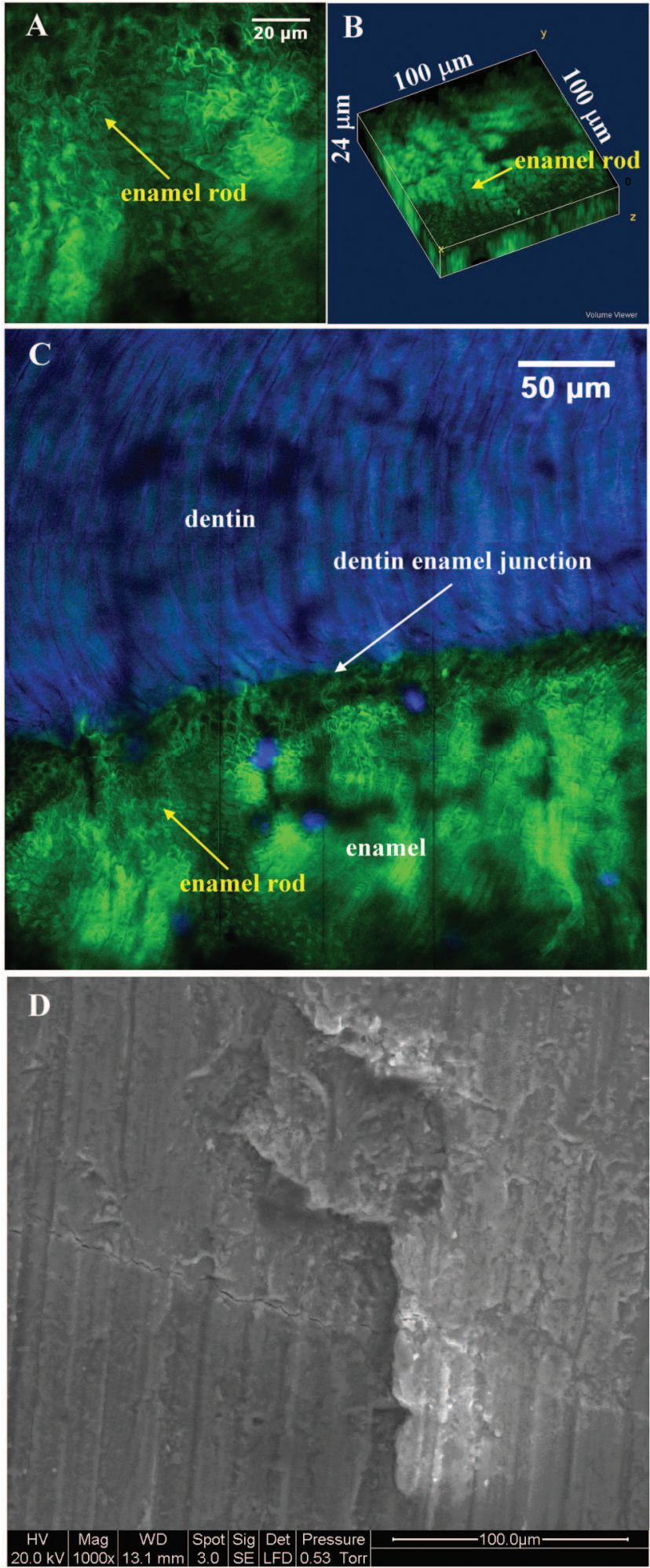

Fig. 2 (A) The AF (green) image of enamel. The arrow points to one of the enamel rods. (B) Three-dimensional projection of the enamel (depth of $24 \mu \mathrm{m}$ ), constructed by sequential axial images acquired $1 \mu \mathrm{m}$ apart. (C) Combined AF and SHG image of the enamel with adjacent dentin. The darker area along the dentin-enamel junction indicates reduced mineralization along that region. (D) SEM image of the tooth's enamel. 

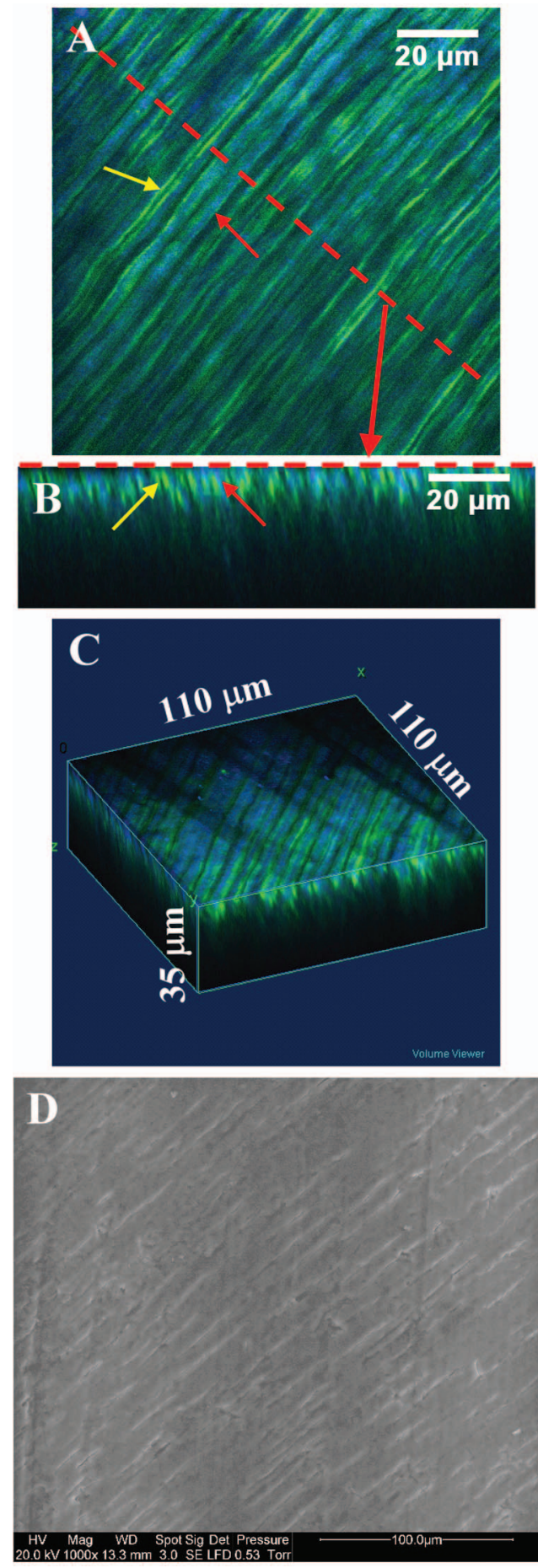

Fig. 3 (A) Multiphoton dentin images showing the combination of multiphoton AF and SHG. The mineralized dentinal tubules, indicated by the yellow arrow, emit AF (green) and SHG (blue) signals, while the collagen fibers of the dentinal structure, indicated by the red arrow, produce mainly a SHG signal. (B) Cross-sectional view of an image stack over the red dashed line area in (A). Yellow and red arrows indicate, respectively, the mineralized and unmineralized parts of dentinal tubules. (C) Three-dimensional projection (depth of $35 \mu \mathrm{m})$ of the middle dentinal area constructed by sequential images taken 1- $\mu \mathrm{m}$ apart. (D) SEM image of the dentin showing the aligned dentinal tubules.
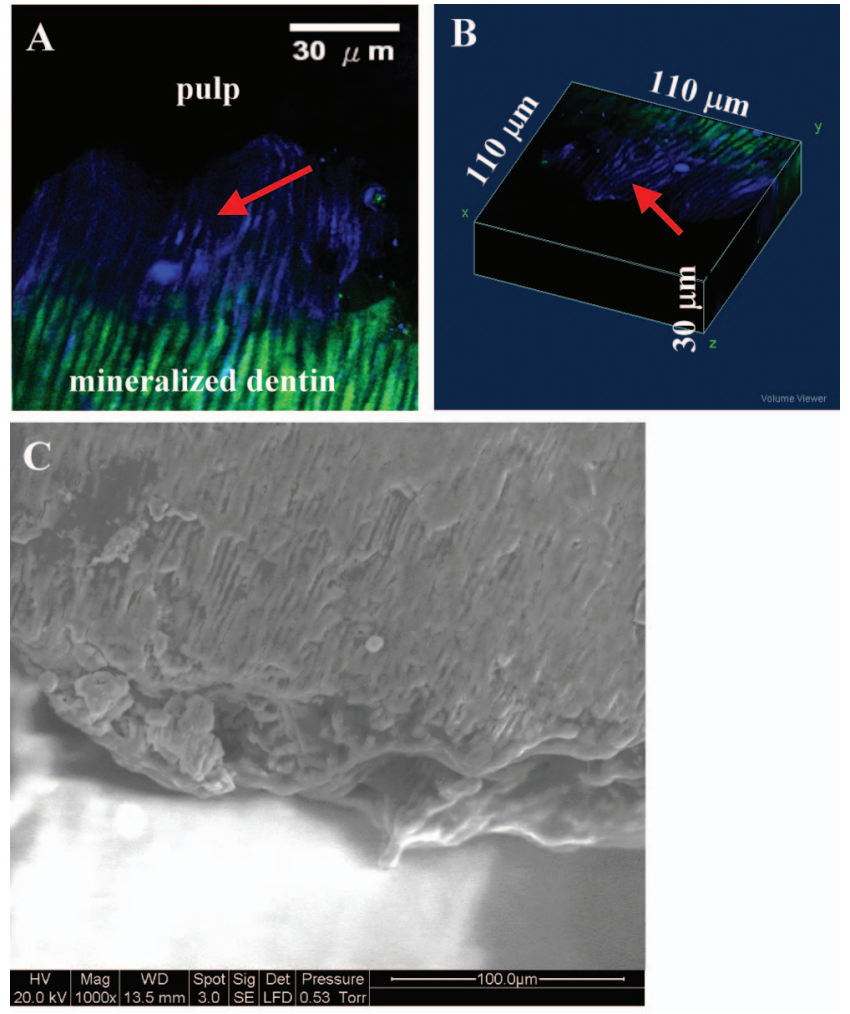

Fig. 4 (A) Combined multiphoton AF (green) and SHG (blue) images of the dentin-pulp junction. The red arrow indicates newly produced circumpulpal dentin, which is unmineralized and produces only the SHG signal. Further away from the pulp, the dentin is mineralized and emits $\mathrm{AF}$ in addition to SHG. (B) Three-dimensional reconstruction of the area shown in (A) using a sequence of 30 images taken $1-\mu \mathrm{m}$ apart. (C) SEM image of the dentin near the pulp demonstrating the curcumpulpal dentin with less regular arrangement of dentinal tubules.

premolar extracted for orthodontic treatment. With the patient's consent and the approval of the Review Committee of College of Medicine at National Taiwan University, we proceeded to prepare the tooth for imaging. First, we cut the tooth along the longitudinal direction with an IsoMet saw (Buehler Ltd., Lake Bluff, Illinois). The tooth specimen is then stored in normal saline solution prior to multiphoton imaging acquisition. In addition to obtaining the multiphoton imaging of the tooth, we also obtained SEM images of the tooth for comparison.

The multiphoton AF and SHG imaging were acquired on a home-built epi-illuminated laser scanning system similar to one described previously by Sun et al. ${ }^{3}$ The system consists of a modified upright microscope (E800, Nikon, Tokyo, Japan) with excitation provided by an $80-\mathrm{MHz}$ femtosecond Tisapphire laser system (Millenia X, Tsunami, Spectra Physics, Mountain View, California) tuned to $780 \mathrm{~nm}$. The raster scanning of the laser excitation is performed by a pair of scanning mirror systems (Model 6220, Cambridge Technology, Cambridge, Massachusetts). The scanning laser beam is then expanded to overfill the back aperture of a water-immersion objective (S FLUOR 40×, numeric aperture 0.8, Nikon). A short pass dichroic mirror (700DCSPXRUV-3p, Chroma Technology, Rockingham, Vermont) was used to separate the 


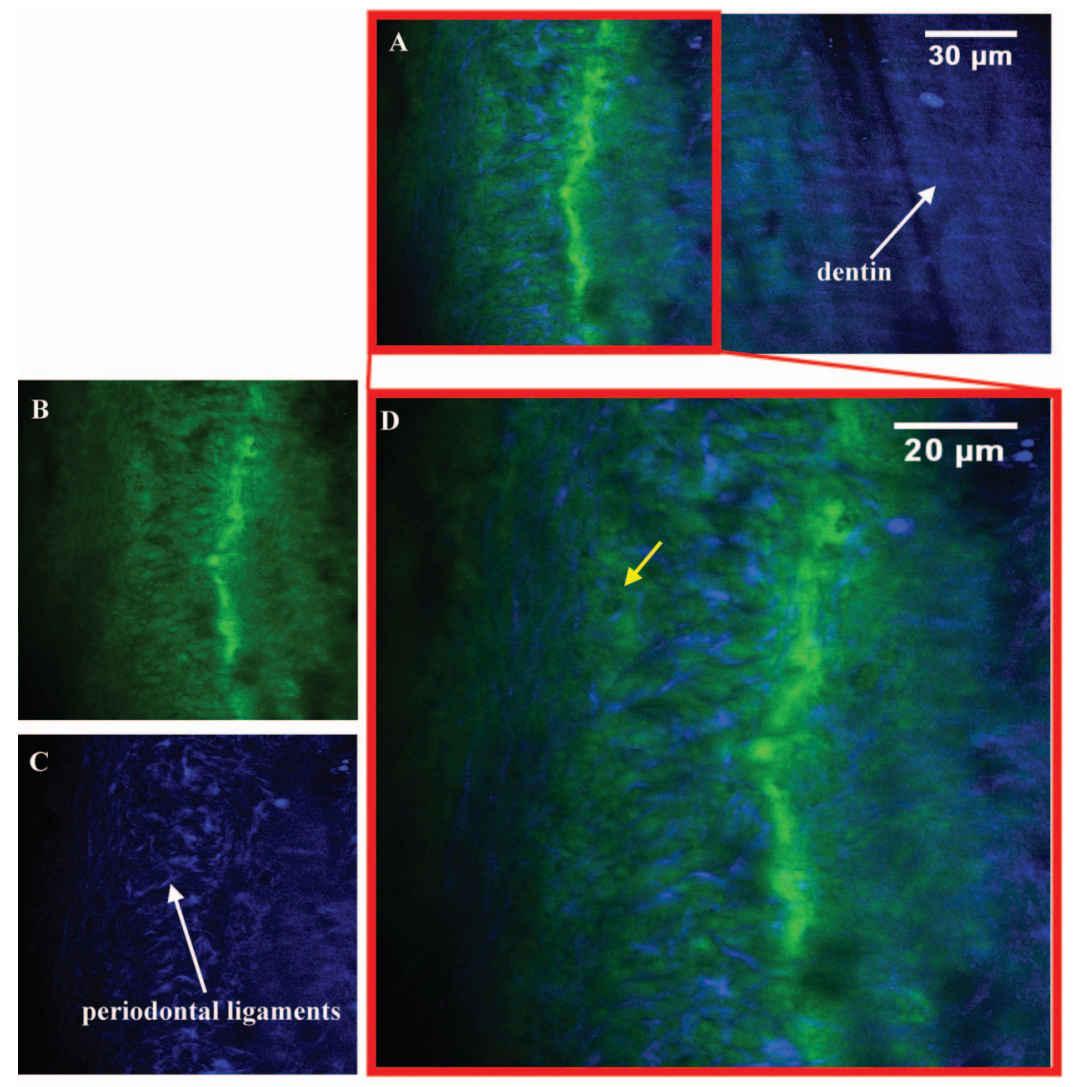

Fig. 5 (A) Large area multiphoton image around the PL. (B) and (C) are the respective AF and SHG images of the selected area of the PL image shown in (A). (D) Enlarged view of the selected region in (A). The yellow arrow points to what is likely to be a fibroblast cell.

excitation and emission. The epi-illuminated multiphoton AF and SHG signals are separated by a long pass dichroic mirror (435DCXR, Chroma Technology) and two bandpass filters (E435LP-E700SP, HQ390/20-2p, Chroma Technology). Each of the two signals is detected with a single-photon counting photomultiplier tube (R7400P, Hamamatsu, Hamamatsu City, Japan), processed by a discriminator, and sent to the computer to form images. The motorized sample stage (H101, Prior Scientific Instruments, Cambridge, United Kingdom) is also controlled by the computer for automated sequential scanning along the $x, y$, and $z$ axes. The sequentially acquired images along the $x$ and $y$ directions in the image plane can be combined to produce large area images, and sequentially acquired images along the axial $z$ axis can be used to construct threedimensional projections of the specimen.

\section{Results}

Figure 1 shows the longitudinal section of the tooth used in this study. The various components of the tooth are labeled in the figure. In the multiphoton image of the enamel (Fig. 2), both the AF and SHG signals were used to characterize the enamel and related structures. In this and subsequent images, we used the green pseudocolor to denote $\mathrm{AF}$ and the blue pseudocolor to represent SHG signal. Figure 2(A) shows a two-dimensional AF image of the enamel, and Fig. 2(B) shows a three-dimensional projection to a depth of $24 \mu \mathrm{m}$. The three-dimensional projection is composed of sequential axial images acquired $1 \mu \mathrm{m}$ apart. These images reveal that
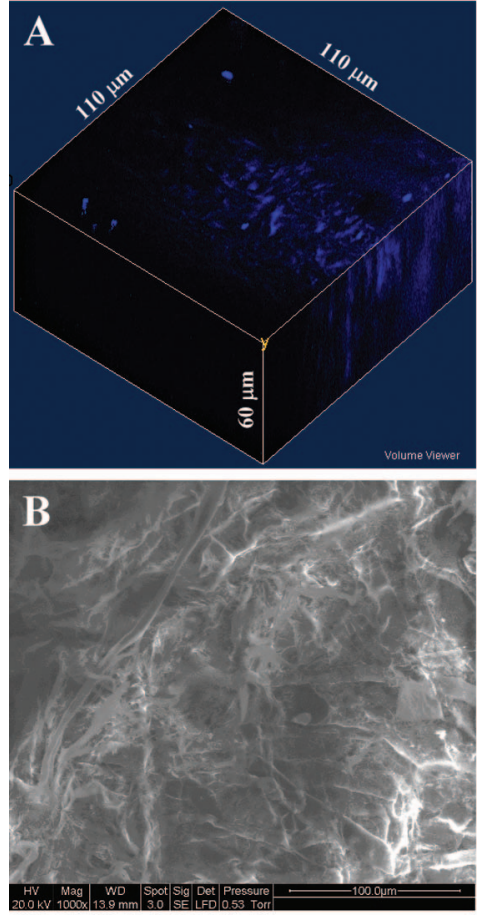

Fig. 6 (A) Three-dimensional reconstruction of the PL from a stack of 60 SHG images acquired $1-\mu \mathrm{m}$ apart. (B) SEM image of the PL showing the ultrastructure of periodontal fibers with cells within the fibrous network. 
two-photon AF imaging is effective in revealing the morphological features of the mineralized enamel rods.

The mineralized enamel rods are formed during the secretory stage by the ameloblasts in the Tomes's process as the cells migrate away from dentin during amelogenesis. The yellow arrows in Figs. 2(A) to 2(C) point to examples of the enamel rods, which also show the parallel nature of the enamel rod aggregates. The dentin-enamel junction is shown in Fig. 2(C), where a darker area approximately $20-\mu \mathrm{m}$ wide corresponds to reduced mineralization along the junction. Although enamel produces mainly strong AF, dentin generates both AF and SHG signals. When compared with the structural motif observed under SEM [Fig. 2(D)], the morphological similarity of the images acquired using the two techniques are evident. The combined two-photon AF and SHG imaging of dentin structure near the enamel-dentin junction is shown in Fig. 3(A), where the structure of the dentinal tubule is visible. The dentinal tubule structure can be better visualized in the sectional image [Fig. 3(B)] and the three-dimensional projection [Fig. 3(C)] constructed from a stack of sequentially acquired axial images acquired 1- $\mu \mathrm{m}$ apart (total thickness of $35 \mu \mathrm{m})$. The collagen composition of the dentinal tubules produces a strong SHG signal (red arrows), while the mineralized part of the dentinal tubules produce both the SHG and AF signals (yellow arrows). Because the mineralized dentin appears to surround each dentinal tubule, it is called peritubular dentin. ${ }^{10}$ By combining the AF and SHG imaging, we can visualize the distribution of the peritubular dentin. As in the case of enamel imaging, comparison shows that multiphoton and SEM imaging [Fig. 3(D)] reveal similar structural architectures of dentin.

We also applied multiphoton microscopy in imaging the dentin region around the pulp area [shown in Fig. 4(A)], and a three-dimensional projection of a $30-\mu \mathrm{m}$ thick image stack acquired at the same position is shown in Fig. 4(B). The newly produced predentins are located just next to the pulp. As shown in Figs. 4(A) and 4(B), these unmineralized predentins (red arrows) produce only the SHG signal with no AF. Further away from the pulp, the dentins are mineralized and can produce AF in addition to the SHG signal. The circumpulpal dentins are incrementally deposited dentin that occurs throughout life. The presence of predentin in our tooth sample shows that the deposition of dentin was an ongoing process for the 30-year-old patient. Continual deposition of dentin is the probable cause for the smaller pulp area shown in Fig. 1. The comparative SEM image of the incrementally deposited dentin structure is shown in Fig. 4(C).

In addition to imaging the enamel and dentin, we also obtained multiphoton images of the PL. Figure 5(A) shows a large area image covering both the dentin and PL. The AF and the SHG of the selected (red square) region is shown respectively in Figs. 5(B) and 5(C). Figure 5(D) is the enlarged view of the selected region of Fig. 5(A). From Fig. 5(D), we see that the fiber structures of the PL can be visualized by SHG imaging. In addition, we also found AF structures within the ligament (yellow arrow) that, most likely, are the fibroblast cells in the ligament. Finally, we acquired and displayed a three-dimensional projection of the PL from a sequence of axially acquired SHG images in Fig. 6(A). Compared to the SEM image [Fig. 6(B)] of the PL, one can see that multiphoton microscopy provides clear three-dimensional structural in- formation of the fiber organization information within the PLs.

In conclusion, we applied and found that multiphoton microscopy can be used to visualize the morphological features of the enamel, dentin, and PL, the three main components of a tooth. We demonstrated that the structure of enamel rods, the mineralized structure of dentinal tubules, and most likely, the cells of the PL can be imaged using two-photon AF signals; whereas the unmineralized structure of dentin and the fiber structure of the PLs can be imaged with the SHG signal. In addition to the ability to obtain morphological information with reduced photodamage and deep sample penetration, multiphoton microscopy can also be combined with polarization resolved methods to obtain structural information below optical resolution. In particular, polarization SHG microscopy has been used to determine the pitch angle of the myosin coil in muscle fiber. ${ }^{15}$ Such technique can be applied to determine submicron structural information for collagen and other tissues that produce SHG signals. This feature and the ability of multiphoton microscopy to visualize the major components of teeth make it a powerful tool for the investigation of dental physiological processes such as tooth regeneration and the diagnosis of tooth pathological conditions.

\section{Acknowledgments}

The authors thank the Core Facility from NTUH Medical Research Center, and the Core Facility of the Institute of Cellular and Organismic Biology, Academia Sinica, for assistance in environmental SEM and the Optical Molecular Imaging Microscopy Core Facility (A5) of the National Research Program for Genomic Medicine, National Science Council, Taiwan for multiphoton image acquisition. We also like to thank National Taiwan University Hospital (NTUH 95-525) and the National Science Council of Taiwan (Grant No. NSC 943112-B-002-015-Y) for financial support.

\section{References}

1. W. Denk, J. H. Strickler, and W. W. Webb, "2-Photon laser scanning fluorescence microscopy," Science 248(4951), 73-76 (1990).

2. P. T. C. So, C. Y. Dong, B. R. Masters, and K. M. Berland, "Twophoton excitation fluorescence microscopy," Аnnu. Rev. Biomed. Eng. 2, 399-429 (2000)

3. Y. Sun, J. W. Su, W. Lo, S. J. Lin, S. H. Jee, and C. Y. Dong, "Multiphoton polarization imaging of the stratum corneum and the dermis in ex-vivo human skin," Opt. Express 11(25), 3377-3384 (2003).

4. S. J. Lin, R. J. Wu, H. Y. Tan, W. Lo, W. C. Lin, T. H. Young, C. J. Hsu, J. S. Chen, S. H. Jee, and C. Y. Dong, "Evaluating cutaneous photoaging by use of multiphoton fluorescence and second-harmonic generation microscopy," Opt. Lett. 30(17), 2275-2277 (2005).

5. H. S. Lee, Y. Liu, H. C. Chen, L. L. Chiou, G. T. Huang, W. Lo, and C. Y. Dong, "Optical biopsy of liver fibrosis by use of multiphoton microscopy," Opt. Lett. 29(22), 2614-2616 (2004).

6. W. R. Zipfel, R. M. Williams, R. Christie, A. Y. Nikitin, B. T. Hyman, and W. W. Webb, "Live tissue intrinsic emission microscopy using multiphoton-excited native fluorescence and second harmonic generation," Proc. Natl. Acad. Sci. U.S.A. 100(12), 7075-7080 (2003).

7. A. Zoumi, A. Yeh, and B. J. Tromberg, "Imaging cells and extracellular matrix in vivo by using second-harmonic generation and twophoton excited fluorescence," Proc. Natl. Acad. Sci. U.S.A. 99(17), 11014-11019 (2002).

8. P. J. Campagnola and L. M. Loew, "Second-harmonic imaging microscopy for visualizing biomolecular arrays in cells, tissues and organisms," Nat. Biotechnol. 21(11), 1356-1360 (2003).

9. E. Brown, T. McKee, E. di Tomaso, A. Pluen, B. Seed, Y. Boucher, 
and R. K. Jain, "Dynamic imaging of collagen and its modulation in tumors in vivo using second-harmonic generation," Nat. Med. 9(6), 796-800 (2003).

10. J. K. Avery, P. F. Steele, and N. Avery, Oral Development and Histology, Thieme Medical Publishers Inc., New York (2001).

11. J. M. Girkin, A. F. Hall, and S. L. Creanor, "Multi-photon imaging of intact dental tissue," in, Proceedings of the 4th Annual Indiana Conference, G. K. Stookey, Ed., Indiana University School of Dentistry, Indianapolis, pp. 155-168 (1999).

12. A. Hall and J. M. Girkin, "A review of potential new diagnostic modalities for Caries lesions," IADR, pp. 89-94 (2004).
13. J. C. Chen, T. C. Yang, and F. J. Kao, "Laser scanning microscopy of enamel and dentin sections with two-photon fluorescence and second harmonic generation," Presented at CLEO/Pacific Rim 2003. The 5th Pacific Rim Conference on Lasers and Electro-Optics, 2003 (2003).

14. N. P. Piesco, "Histology of dentin," in Oral Development and Histology, pp. 242-261, Thieme Medical Publishers, New York (1994).

15. S. V. Plotnikov, A. C. Millard, P. J. Campagnola, and W. A. Mohler, "Characterization of the myosin-based source for second-harmonic generation from muscle sarcomeres," Biophys. J. 90(2), 693-703 (2006). 